\title{
ASSEMBLY OF OSSEOUS FRAGMENTS IN ORTHOPAEDIC SURGERY: THE NEED FOR NEW STANDARDS OF EVALUATION
}

\author{
Olivier Cartiaux ${ }^{1}$, Laurent Paul ${ }^{2}$, Pierre-Louis Docquier ${ }^{2}$, Xavier Banse ${ }^{2}$, Benoit \\ Raucent'
}

1Department of Mechanical Engineering, Université catholique de Louvain, 2 Place du Levant, 1348 Louvain-la-Neuve, Belgium

2Department of Orthopaedic Surgery, Cliniques universitaires St-Luc, Université catholique de Louvain, 10 Avenue Hippocrate, 1200 Brussels, Belgium

olivier.cartiaux@uclouvain.be

\begin{abstract}
In orthopaedic surgery, intra-operative bone machining and assembly of osseous fragments are two very important research areas. One of the most challenging applications is the treatment of malignant osseous tumors within the pelvis due to its complex tri-dimensional geometry. The conventional surgical procedure includes tumoral resection (cutting of the osseous tumor) and reconstruction by allograft (assembly of fragments). Accuracy of bone cutting and osseous assembly has not yet been documented. This paper presents an experimental study on plastic bones, with experienced surgeons working under ideal conditions. The goal was to assess the accuracy by using geometrical parameters resulting from the surgical usual language: surgical margin for tumor cuttings, and maximal gap, gap volume and mean gap between the 2 osseous fragments. Both mean values and correlation between assembly parameters were relatively poor. Experienced surgeons did not manage to consistently perform accurate cuttings and osseous assemblies, even under ideal working conditions. The complex tri-dimensional architecture of the pelvis can mainly explain this inaccuracy. There is a need to adapt computer and robotic assisted technologies for tumor cuttings and osseous assemblies. Finally, our attempt to evaluate accuracy using simple geometrical parameters, was not satisfactory. There is a need to define new evaluation standards for these assemblies. We think that mechanical engineering tools like geometrical tolerances and mechanical fittings are more suitable for this problem of quality evaluation.
\end{abstract}




\section{Introduction}

In orthopaedic surgery, intra-operative bone machining and assembly of osseous fragments are two very important research areas. One of the most challenging applications is the treatment of malignant osseous tumors within the pelvis. It is due to the complex tri-dimensional (3D) geometry of the bony pelvis and the proximity of organs and structures difficult to reach, like vessels, bladder, rectum, sciatic nerve [1]. Nowadays, conventional surgical procedure includes tumoral resection (cutting of the tumor), using an oscillating saw, with an adequate margin [2-4].

Most usual available data to deal with this problem are clinical post-operative outcomes. Enneking [5] described a classification of surgical margin as radical, wide, marginal or intralesional. Recurrence is the major post-operative complication. Local recurrence rates from 28 to $35 \%$ were reported concerning limb-salvage procedures [6]. However, to our knowledge, geometrical accuracy of bone tumor cutting has not yet been experimentally documented.

Several osseous reconstruction methods exist: autograft (taken on the patient himself), allograft (taken on cadaver), or customised prosthesis. Whatever the used technique, surgeons have to perform assemblies of osseous fragments. Osseous massive allografts present some technical advantages: they can be shaped to perfectly fit the pelvic defect, they allow good reinsertion of soft tissues like tendons and muscles, and they provide true anatomical restoration of the complex $3 \mathrm{D}$ architecture of the pelvis [6].

Again, most usual available data are clinical rates, like infection, non-union and fracture of the reconstruction. Many studies have dealt with these post-operative outcomes [7-16]. The main information is the need for an accurate and intimate contact at the host-graft junction (the cutting site) in order to promote and accelerate union [17]. However, ranges of all the reported rates are large. Assessing the quality of a pelvic reconstruction, i.e. an osseous assembly, using clinical outcomes is still a difficult task.

In this study, we designed an experimental model on plastic pelvic bones to simulate the conventional (free-hand) procedure of treatment of osseous tumors. We tried to assess the geometrical accuracy of the surgical gesture, based on geometrical parameters resulting from the surgical usual language.

\section{Material and methods}

4 experienced senior surgeons (S1, S2, S3, S4), used to pelvic surgery [6], were each asked to perform cutting of 3 different pelvic tumors and their reconstruction. 12 identical plastic pelves and 12 plastic left hemipelves were procured (Sawbones 
Worlwide, Pacific Research Laboratories Inc., Vashon, WA) and considered as host bones (host) and allografts (graft) respectively.

\subsection{Conventional surgical procedure: design of an experimental model}

\subsubsection{Virtual tumors}

The 12 hosts were scanned (spiral Elscint Twin CT scanner with $2.7 \mathrm{~mm}$ slice thickness). 3 sets of tumor were virtually created : tumor 1 in zone I (Tl, iliac wing), tumor 2 in zone II (T2, acetabulum), and tumor 3 over zones II and III (T3, acetabulum and obturator foramen) according to Enneking [18]. The tumor was represented by a sphere of fixed diameter and placed on the 3D CT-scan of the pelvis (Figure 1A) using the visualization software Volview, version 2.0.5 (Kitware Inc., New-York, USA).

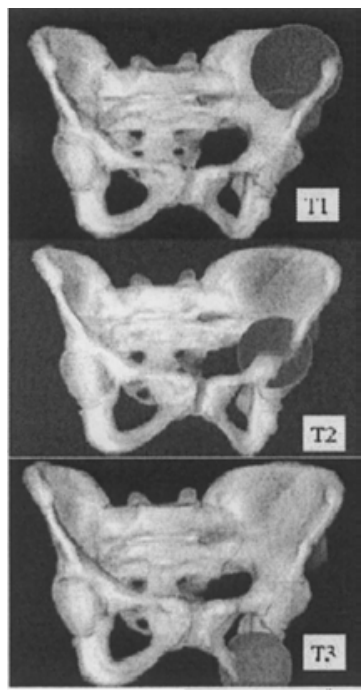

A

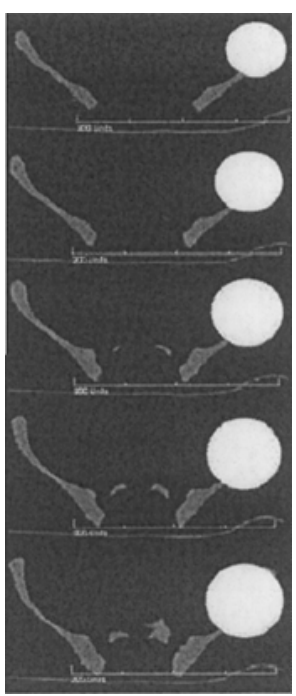

$\mathbf{B}$

Fig. 1. Virtual tumors. A) 3D views : T1 in zone I of Enneking, T2 in zone II and T3 in zone II-III. B) Examples of transversal slices, given to surgeons, of the virtual tumor in zone I (T1) for cutting planning. A graduated scale of $200 \mathrm{~mm}$ was given for each slice 


\subsubsection{Tumor cutting}

Each surgeon received a print with 32 sequential coronal slices, 32 transversal slices and 20 sagittal slices for each tumor (Figure 1B). A graduated scale of $200 \mathrm{~mm}$ was available on each slice.

The surgeon could plan resection planes and perform cutting of the 3 tumors (T1, T2, T3) without limitation in time. Instruction was given to respect a $10 \mathrm{~mm}$-surgical margin with an accepted tolerance of $5 \mathrm{~mm}$ above or below. In other words, surgeon had to perform resection within the interval of tolerance $[5 \mathrm{~mm} ; 15 \mathrm{~mm}]$. Based on the 2D slices of the tumor, he could note some landmarks with a skin marker on the host to guide the cutting. After planning and landmarking, he had to perform tumor resection by cutting the host using an oscillating saw (Howmedica Chirodrill 1200 set, Germany). In order to simulate patient positioning on the operating table, the host was rigidly fixed by a steel clamp with a $360^{\circ}$-rotational base (Figure 2 ).

\subsubsection{Host-Graft assembly}

Each surgeon had to reconstruct the pelvis, after each tumor cutting. An oversized graft was given to reconstruct $\mathrm{T} 1$ and $\mathrm{T} 3$ in order to increase the technical difficulties. A sizematched graft was chosen to reconstruct $\mathrm{T} 2$ because it was simulating an articular reconstruction. As for tumor resection, surgeon could note some landmarks with a skin marker on the graft to guide the cutting. He could also place the graft into the gap to check its size. Additional cuttings were allowed to each surgeon to optimize the graft cutting. The host-graft reconstruction was temporarily fixed with K-wires $(2 \mathrm{~mm}$ diameter).

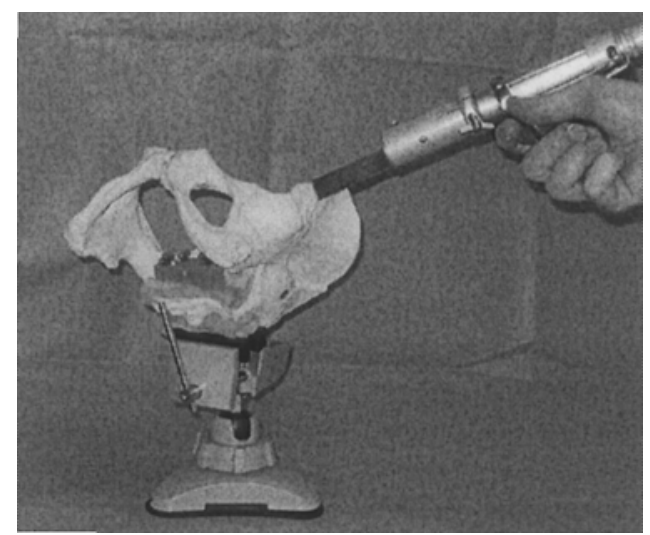

Fig. 2. The sawbone is rigidly fixed by a steel clamp with a $360^{\circ}$-rotational base. The cutting is performed using an oscillating saw. 


\subsection{Evaluation of tumor cuttings}

After tumor cuttings, resected parts were scanned (spiral Elscint Twin CT scanner with $2.7 \mathrm{~mm}$ slice thickness) and registered with the 3D CT-scan of the corresponding host. Margin error was calculated as the minimal distance between the $10 \mathrm{~mm}$-surgical margin and each cutting plane, using Volview and its distance measurement tool. Negative values of margin error were given for cutting below the target and positive values for cutting above the target. This allowed verifying if the interval of tolerance [ 5 $\mathrm{mm} ; 15 \mathrm{~mm}$ ] had been respected or not.

\subsection{Evaluation of host-graft assemblies}

Each host-graft junction (HGJ) was evaluated by 4 different methods, each one qualifying a geometrical parameter usually used by surgeons.

3 different observers classified each HGJ according to the degree of contact between host and graft (ordered categorical classification): degree 1 for full contact, 2 for contact $>50 \%, 3$ for contact $<50 \%$ or 4 for no contact.

Maximal gap between host and graft of each HGJ was measured with an electronic caliper (CD-15CP, Mitutoyo Inc., Aurora, IL) by the 3 same observers.

Gap volume between host and graft was measured for all the HGJ. An epoxy paste with scanner-high-density (pc-7 Heavy Duty Paste Epoxy, PC-products, Allentown, USA) was used to completely fill the gap of the HGJ. All the constructs were scanned (spiral Elscint Twin CT scanner with $2.7 \mathrm{~mm}$ slice thickness). As the density of the paste was high and very different from the Sawbone, a threshold segmentation permitted the elimination of voxels corresponding to the Sawbone (Figure 3). The number of voxels corresponding to the paste was calculated, giving the HGJ gap volume.

Finally, contact surface available on the host was measured for each HGJ. Mean gap between host and graft was then calculated for each HGJ as the ratio of gap volume to the corresponding contact surface. 
A

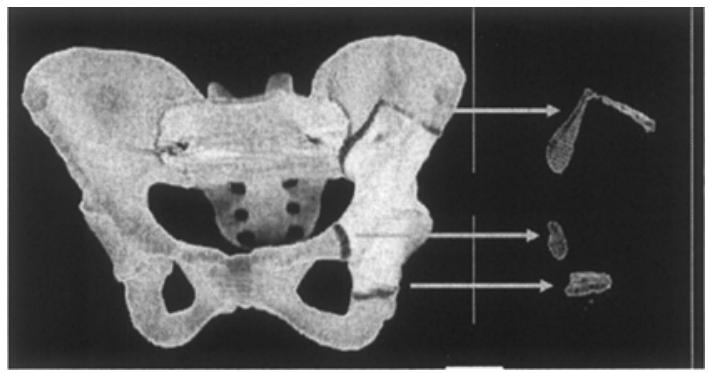

B

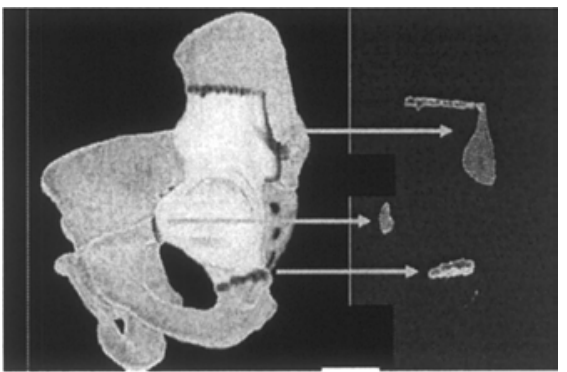

Fig. 3. 3D CT-scan of a host-graft reconstruction with the scanner-high-density epoxy paste filling gaps. A threshold segmentation permitted to eliminate voxels corresponding to the Sawbone and to calculate gap volume of the host-graft junction. A) Antero-posterior view before and after segmentation of the paste. B) Lateral view.

\subsection{Statistics}

All analyses were performed using statistical software SPSS $®$, version 12.0 (SPSS Inc., Chicago, IL. The inter-observer agreement of the variable "degree of contact" was calculated using weighted kappa statistics [19,20]. Non parametric Mann-Whitney tests or non parametric Wilcoxon tests were performed to compare each pair of observers for the variable "maximal gap" and each pair of surgeons for the 4 variables "margin error", "maximal gap", "gap volume" and "mean gap". Differences were considered statistically significant when $p<0.05$. To assess linear relationship between the 4 HGJ variables ("degree of contact", "maximal gap", "gap volume" and "mean gap"), square of Pearson correlation coefficient was measured. 


\section{Results}

24 cutting planes and 24 HGJ were available for evaluation: 6 cutting planes and HGJ for each surgeon ( 1 cutting plane and HGJ for each T1, 3 for each T2 and 2 for each T3).

\subsection{Tumor cuttings}

Mean margin error was 5.27 (SD 4.42) mm. Margin errors of the 4 surgeons were not statistically significantly different (Table 1). Among the 24 cutting planes, 11 did not respect the accepted $5 \mathrm{~mm}$-tolerance above or below the target (Figure 4). 2 cutting planes were intralesional: error of $15.02 \mathrm{~mm}$ and $15.14 \mathrm{~mm}$ below the target respectively.

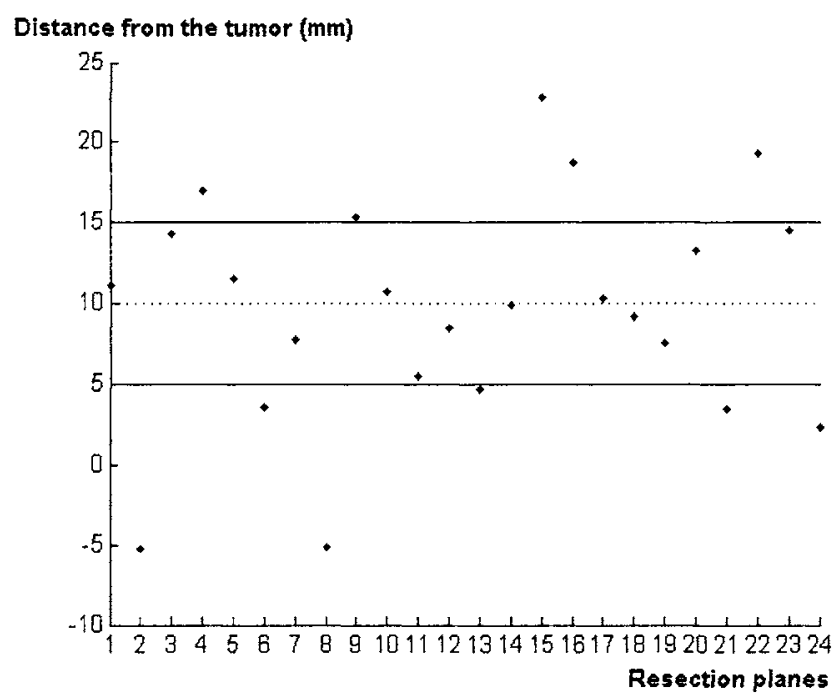

Fig. 4. Distances between the 24 cutting planes and the tumor. The dashed line represents the recommended $10 \mathrm{~mm}$-safe margin, and the lines above and below it represent the accepted tolerance interval. 


\subsection{Host-Graft assemblies}

Of the 24 HGJ, 22 were identically classified by the 3 observers (Obs1, Obs2 and Obs3). Most of HGJ were classified " $<50 \%$ " contact (Figure 5c). 2 HGJ were classified differently: the first was classified " $>50 \%$ " contact by 1 observer and " $<50 \%$ " contact by the 2 others, and the second was classified " $>50 \%$ " by 2 observers and " $<50 \%$ " by one observer. Weighted Kappa correlation coefficients between observers were always very good: $\kappa=0.91$ for (Obs1, Obs2) and (Obs1, Obs3) and $\kappa=1.0$ for (Obs2, Obs3).

Mean maximal gap between host and graft for the $24 \mathrm{HGJ}$ was 3.31 (SD 1.93) mm. Differences between surgeons were not statistically significant (Table 1). Measurements performed by the 3 observers were highly correlated: Spearman correlation coefficient was 0.95 for (Obs1, Obs2), 0.96 for (Obs1, Obs3) and 0.97 for (Obs2, Obs3). Differences between observers were statistically significant for (Obs 1, Obs 3$)(p=0.03)$ and for (Obs2, Obs3) ( $p=0.02)$.

Mean gap volume between host and graft was $2.60\left(\right.$ SD 2.18) $\mathrm{cm}^{3}$. There was no statistically significant difference between surgeons (Table 1).

Gap volume correlated strongly with contact surface (Figure 5a). Mean gap between host and graft was 3.22 (SD 2.08) $\mathrm{mm}$. There was no statistically significant difference between surgeons, except between Surg1 and Surg2 (Table 1).

Correlation between the $4 \mathrm{HGJ}$ variables was poor (Figure 5). Squared Pearson correlation coefficient $r^{2}$ was 0.26 between maximal gap and gap volume, 0.19 between maximal gap and mean gap and 0.05 between gap volume and mean gap.

Table 1. Margin error and host-graft junction parameters according to the 4 surgeons (mean \pm SD)

\begin{tabular}{|c|c|c|c|c|}
\hline & \multirow{2}{*}{$\frac{\text { Tumor cutting }}{\text { Margin error (mm) }}$} & \multicolumn{3}{|c|}{ Host-Graft assembly } \\
\hline & & Maximal gap (mm) & Gap volume $\left(\mathrm{cm}^{3}\right)$ & Mean gap $(\mathrm{mm})$ \\
\hline \multicolumn{5}{|c|}{ Surgeon } \\
\hline S1 & $5.90 \pm 5.09$ & $4.22 \pm 2.63$ & $2.58 \pm 2.84$ & $2.39 \pm 0.71^{a}$ \\
\hline \$2 & $4.89 \pm 5.32$ & $2.98 \pm 0.94$ & $2.98 \pm 2.09$ & $3.92 \pm 0.79^{a}$ \\
\hline S3 & $4.67 \pm 5.24$ & $2.43 \pm 1.42$ & $2.17 \pm 2.33$ & $2.51 \pm 1.43$ \\
\hline S4 & $5.63 \pm 2.65$ & $3.63 \pm 2.25$ & $2.67 \pm 1.88$ & $4.08 \pm 3.72$ \\
\hline
\end{tabular}

"Mann-Whitney $U$ test $p=0.016$ 


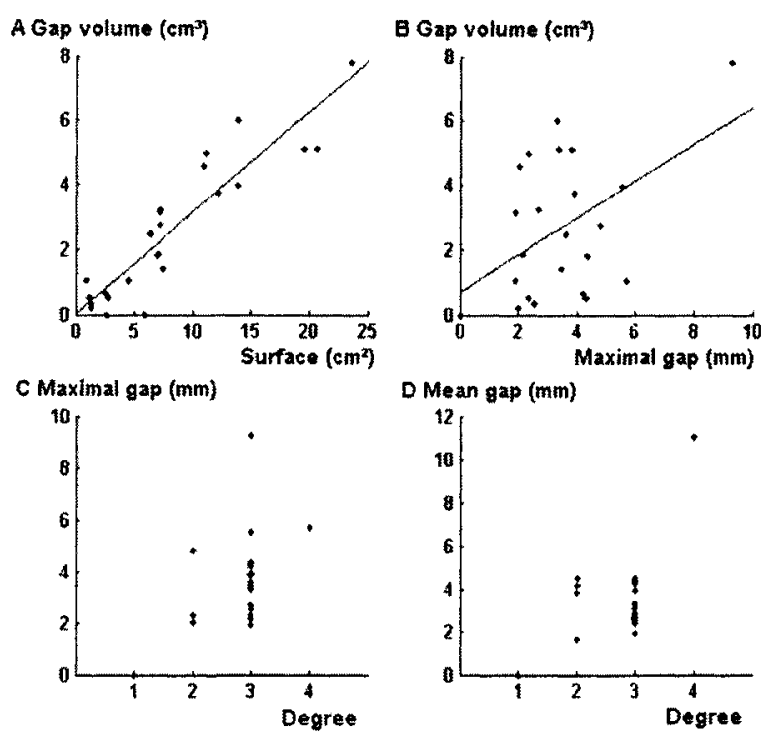

Fig. 5. Correlation between host-graft junction parameters : maximal gap, gap volume, contact surface, mean gap and degree of contact.

\section{Discussion}

This study was performed on plastic pelves under ideal working conditions: complete visualisation of the bone surface, complete accessibility, absence of muscles, nerves and bleeding. Figure 4 showed that 4 experienced surgeons did not manage to consistently respect a $5 \mathrm{~mm}$-tolerance above or below a requested $10 \mathrm{~mm}$-surgical margin. Moreover, there was a great variation among results of each surgeon, demonstrating the lack of accuracy.

According to the classification of surgical margin made by Enneking [5], Delloye [6] in their series of 24 patients, reported that surgical resection was wide in 19 cases, marginal in 6 and intralesional in 1. Our data, obtained under ideal working conditions, are consistent with those findings.

Inaccuracy during tumor resection can thus be explained by the anatomy of the pelvis. Due to its complex 3D geometry, delineating tumor extension, planning cutting planes on 2D CT slices, manually transferring this planning on the bone and performing the resection are difficult tasks even for experienced surgeons.

We believe that the accuracy of tumor cutting could be improved by using computer and/or robotically assisted (CA) technologies. Optical navigation systems using 
preoperative CT-scan data already exist for tumor resection in the pelvis $[21,22]$ and also for sacroiliac screw insertion [23], pelvic osteotomies [24,25] or pelvic ring fracture reduction [26,27]. However, to our knowledge, a comparison of accuracy of osseous pelvic tumor resection between conventional and CA procedures has not yet been performed. Data presented in this study could be useful for further evaluation of CA technologies.

We explored several methods to quantify specific geometrical properties of a HGJ. Classification of HGJ according to the degree of contact and measurement of maximal gap between host and graft offered good repeatability. For maximal gap, Obs3 had tendency to measure smaller gaps than Obs1 and Obs2, resulting in a significant difference. However, mean difference was about $10 \%$ of the mean maximal gap.

Enneking [17] emphasised the need for an accurate and intimate contact at the osteotomy site in order to promote and accelerate union. Delloye [6] stated gaps and narrow surfaces were both concerns of non-union at the junctions. Consequently, we assumed a good reconstruction, i.e. a good osseous assembly, has a good degree of contact, a small maximal gap, a small gap volume and a small mean gap. However, correlation between our HGJ measures was relatively poor.

Gap volume has some clinical meaning. It quantifies osteogenesis required to completely fill the gap between host and graft. Gap volume was poorly correlated to maximal gap (Figure 5b) and degree of contact. Junctions with smallest gap volumes had not necessarily smallest maximal gaps nor highest degrees of contact.

In conclusion, the lack of correlation and the great variation among each result show that assessing the quality of an osseous assembly by our HGJ geometrical parameters is not efficient. It is also impossible to point the different sources of error that can occur during reconstruction: manual transfer of cutting planes on the allograft, cutting of the allograft, positioning and orientation of the cutting tool on the bone, etc. As for tumor cutting, we believe that using CA technologies could improve the accuracy of allograft cutting.

Even for experienced surgeons working under ideal conditions, our attempt to define what a good pelvic reconstruction is was not satisfactory. Again, this can mainly be explained by the complex 3D geometry of the pelvis and consequently by the great diversity of encountered HGJ. We believe that there is a need to define new standards of evaluation that would be independent of the bony structure.

We think that mechanical engineering tools, like geometrical tolerances and mechanical fittings, could be well adapted to this problem of evaluation of an osseous assembly. Well-known concepts like flatness, roughness, parallelism, angularity, etc, or new concepts like modal tolerancing [28] seem to be interesting tools. New research works are about to be launched to know how these tools could be used to evaluate the geometrical accuracy of bone machining and osseous assembly and how they could be integrated in the usual surgical language. 


\section{Acknowledgments}

The authors would like to thank Pr Christian Delloye and Dr Olivier Cornu of the Department of Orthopaedic Surgery (Cliniques universitaires St-Luc, Université catholique de Louvain, Belgium) for performing the simulated surgeries.

\section{References}

1. D. Donati, A. El Ghoneimy, F. Bertoni, C. Di Bella and M. Mercuri, Surgical treatment and outcome of conventional pelvic chondrosarcoma, J Bone Joint Surg (Br) 87, 1527-1530 (2005).

2. N. Kawaguchi, S. Matumoto and J. Manabe, New method of evaluating the surgical margin and safety margin for musculoskeletal sarcoma, analysed on the basis of 457 surgical cases, $J$ Cancer Res Clin Oncol 121, 555-563 (1995).

3. M.E. Pring, K.L. Weber, K. Unni and F.H. Sim, Chondrosarcoma of the pelvis : A review of sixty-four cases, J Bone Joint Surg (Am) 83, 1630-1642 (2001).

4. K.L. Weber, M.E. Pring and F.H. Sim, Treatment and outcome of recurrent pelvic chondrosarcoma, Clin Orthop 397, 19-28 (2002).

5. W.F. Enneking, S.S. Spanier and M.A. Goodman, Current concepts review. The surgical staging of musculoskeletal sarcoma, J Bone Joint Surg (Am) 62, 1027-1030 (1980).

6. C. Delloye, X. Banse, B. Brichard, PL. Docquier and O. Cornu, Pelvic reconstruction with a structural pelvic allograft after resection of a malignant bone tumor, J Bone Joint Surg (Am) 89, 5795-87 (2007).

7. R.S. Bell, A.M. Davis, J.S. Wunder, T. Buconjic, B. McGoveran and A.E. Gross, Allograft reconstruction of the acetabulum after resection of stage-IIB sarcoma. Intermediate-term results, $J$ Bone Joint Surg (Am) 79, 1663-1674 (1997).

8. N. Dion and F.H. Sim, The use of allografts in orthopaedic surgery. Part I : The use of allografts in musculoskeletal oncology, $J$ Bone Joint Surg (Am) 84, 644-654 (2002).

9. G.E. Friedlaender, D.M. Strong, W.W. Tomford and H.J. Mankin, Long-term follow-up of patients with osteochondral allografts. A correlation between immunologic responses and clinical outcome, Orthop Clin North Am 30, 583-588 (1999).

10. A. Hillman, C. Hoffman, G. Gosheger, R. Rödl, W. Winkelmann and T. Ozaki, Tumor of the pelvis : complications after reconstruction, Acta Orthop Trauma Surg 123, 340-344 (2003).

11. F.J. Hornicek, M.C. Gebhardt, W.W. Tomford, J.I. Sorger, M. Zavatta, J.P. Menzner and H.J. Mankin, Factors affecting nonunion of the allograft-host junction, Clin Orthop Relat Res 382, 87 $98(2001)$.

12. H.J. Mankin, F.J. Hornicek and K.A. Raskin, Infection in massive bone allografts, Clin Orthop Relat Res 432, 210-216 (2005).

13. E. Ortiz-Cruz, M.C. Gebhardt, L.C. Jennings, D.S. Springfield and H.J. Mankin, The results of transplantation of intercalary allografts after resection of tumors, J Bone Joint Surg (Am) 79 , 97-106 (1997).

14. J.L. Sorger, F.J. Hornicek, M. Zavatta, J.P. Menzner, M.C. Gebhardt, W.W. Tomford and H.J. Mankin, Allograft fractures revisited, Clin Orthop 382, 66-74 (2001).

15. R.C. Thompson Jr., A. Garg, D.R. Clohisy and E.Y. Cheng, Fractures in large-segment allografts, Clin Orthop Relat Res 370, 227-235 (2000). 
16. D.L. Wheeler and W.F. Enneking, Allograft bone decreases in strength in vivo over time, Clin Orthop Relat Res 435, 36-42 (2005).

17. W.F. Enneking and D.A. Campanacci, Retrieved human allografts. A clinicopathological study, $J$ Bone Joint Surg (Am) 83, 971-986 (2001).

18. W.F. Enneking and W.K. Dunham, Resection and reconstruction for primary neoplasms involving the innominate bone, $J$ Bone Joint Surg ( $\mathrm{Am})$ 60, 731-746 (1978).

19. A. Petrie, Stastitics in orthopaedic papers, J Bone Joint Surg (Br) 88, 1121-1136 (2006).

20. J.R. Landis and G.G. Koch, The measurement of observer agreement for categorical data, Biometrics 33, 159-174 (1977).

21. T. Hüfner, M. Kfuri Jr., M. Galanski, L. Bastian, M. Loss, T. Pohlemann and C. Krettek, New indications for computer-assisted surgery: tumor resection in the pelvis, Clin Orthop Relat Res 426, 219-225 (2004).

22. C. Krettek, J. Geerling, L. Bastian, M. Citak, F. Rucker, D. Kendoff and T. Hüfner, Computer aided tumor resection in the pelvis, Injury 35, 79-83 (2004).

23. E. Gautier, R. Bachler, P.F. Heini and L.P. Nolte, Accuracy of computer-guided screw fixation of the sacroiliac joint, Clin Orthop Relat Res 393, 310-317 (2001).

24. F. Langlotz, R. Bächler, U. Berlemann, L.P. Nolte and R. Ganz, Computer assistance for pelvic osteotomies, Clin Orthop Relat Res 354, 92-102 (1998).

25. G. van Hellemondt, M. de Kleuver, A. Kerckhaert, P. Anderson, F. Langlotz, L.P. Nolte and P. Pavlov, Computer-assisted pelvic surgery: an in vitro study of two registration protocols, Clin Orthop Relat Res 405, 287-293 (2002).

26. T. Hüfner, T. Pohlemann, S. Tarte, A. Gänsslen, M. Citak, N. Bazak, U. Culemann, L.P. Nolte and C. Krettek, Computer-assisted fracture reduction: novel method for analysis of accuracy, Comput Aided Surg 6, 153-159 (2001).

27. T. Hüfner, T. Pohlemann, S. Tarte, A. Gänsslen, J. Geerling, N. Bazak, M. Citak, L.P. Nolte and C. Krettek, Computer-assisted fracture reduction of pelvic ring fractures: an in vitro study, Clin Orthop Relat Res 399, 231-239 (2002).

28. PA. Adragna, S. Samper, H. Favrelière, Tolérancement modal - un langage de spécification, $2^{\text {nde }}$ journée européenne du tolérancement, Annecy (June 20, 2006). 